\title{
Estimation du tauX de prévalence de L'HYDATIDOSE bOVINe en Midi-Pyrénées
}

\author{
BICHET H.* \& DORCHIES P.**
}

Summary : Prevalence of hydatidosis in CATtLe in Midi-Pyrénées, FRANCE

Since 1994 " Réseau VEGA * (veterinary survey network) has organized a record of sanitary information in 14 slaughterhouses in the Midi-Pyrénées. Data about hydatidosis in cattle are centralized, analysed, then sent namely to each stockbreeder concerned. Estimation of the prevalence rate from 1994 to 1996 is $0.28 \%$ for animals and $2.5 \%$ for livestock. A marked decrease of rates was noticed during this three-year monitoring period. Nevertheless, the Pyrenean area remains more affected than the North of the region. In an outbreak of hydatidosis, a few animals are carriers. Bovine infestation must be considered as revealing a rural cycle. Moreover, the link between bovine hydatidosis and ovine transhumance seems to be confirmed. Using livestock as epidemiological units is innovative in terms of hydatidosis. This approach allows a better adjustment of parasitism control and introduces the notions of the outbreak and the risk of human contamination. In the Midi-Pyrénées region, local human cases of hydatisosis are few. However, the absence of compulsory notification and of databases, on the one hand, and the extreme difficulty of confirming the autochthonous nature of the contamination, on the other hand, limit the reliability of data. A better collaboration between physicians and veterinarians would reduce animal prevalence and the risk of human contamination.

KEY WORDS : hydatidosis in cattle, Echinococcus granulosus, prevalence, evolution, South-west of France.

\section{INTRODUCTION}

L 'échinococcose-hydatidose est une zoonose parasitaire sévissant en de très nombreux pays, tout particulièrement sur le pourtour méditerranéen (Charnine, 1986; Cruz Paiz, 1991; Gusbi, 1990; Mate, 1991; Navarrete, 1991; Euzeby, 1997). Elle est devenu progressivement ubiquitaire s'étendant à des zones urbaines jusqu'alors épargnées.

De nombreuses enquêtes ont été menées à travers le monde afin d'estimer l'importance et la répartition de

\footnotetext{
* Réseau VEGA, rue de Las Escoumes, BP 83, F-09007 Foix Cedex. ** École Vétérinaire de Toulouse, 23, chemin des Capelles, F-31076 Toulouse Cedex.

Correspondance : Hervé Bichet.

Tél. : 0561021630 - Fax : 0561021620.
}

\section{Résumé :}

Depuis 1994 une collecte d'informations sanitaires est organisée par le Réseau VEGA (réseau vétérinaire d'épidémiosurveillance) dans 14 abattoirs de Midi-Pyrénées. Les données de saisies pour hydatidose bovine sont centralisées, analysées puis communiquées nominativement aux éleveurs concernés. L'estimation du taux de prévalence pour la période 1994-1996 est de 0,28\% pour les animaux et 2,5\% pour les cheptels. Une diminution sensible de ces taux a été enregistrée au cours des trois années de surveillance. Les départements pyrénéens restent néanmoins plus touchés que le nord de la région. Au sein des foyers d'hydatidose, le nombre d'animaux atteints est faible. L'infestation bovine doit être considérée comme révélatrice d'un cycle rural. Par ailleurs, le lien entre hydatidose bovine et transhumance ovine semble se confirmer. L'utilisation du cheptel en tant qu'unité épidémiologique est originale en matière d'hydatidose. Cette approche permet de mieux orienter la lutte antiparasitaire et introduit les notions de foyer et de risque de contamination humaine. Dans la région, le nombre de cas autochtones d'hydatidose chez l'homme est faible. Cependant, l'absence de déclaration obligatoire et de centralisation des informations d'une part et l'extrême difficulté de confirmer le caractère autochtone de la contamination d'autre part limitent la fiabilité de ces résultats. Une collaboration plus étroite des médecins et des vétérinaires serait de nature à réduire la prévalence animale et le risque de contamination humaine.

MOTS CLÉS : hydatidose bovine, Echinococcus granulosus, prévalence, évolution, Sud-Ouest de la France.

l'hydatidose. Néanmoins, la notion de cheptel en tant qu'unité épidémiologique n'est jamais prise en compte. Depuis 1994, le Réseau vétérinaire d'épidémiosurveillance globale appliquée (Réseau VEGA) de la région Midi-Pyrénées organise une collecte en abattoir, sur les bovins. Les données ainsi recueillies sont centralisées, analysées puis restituées aux détenteurs d'animaux atteints. Un conseil technique accompagne ces résultats incitant les propriétaires à vermifuger leurs chiens.

En l'absence d'identification fiable et du fait de la complexité des circuits commerciaux, l'étude n'a pu être menée dans l'espèce ovine. La connaissance précise de l'origine des bovins abattus permet d'estimer le taux de prévalence de l'hydatidose à $0,28 \%$ des animaux et $2,5 \%$ des cheptels, en Midi-Pyrénées, pour la période 1994-1996. 


\section{MATÉRIEL ET MÉTHODES}

\section{ABATTOIRS}

L

es données ont été recueillies par les services d'inspection vétérinaire en abattoir, dans l'ensemble des 14 sites d'observation de la région Midi-Pyrénées (Fig. 1). Tous les abattoirs sollicités ont accepté d'effectuer cette collecte d'informations sanitaires. Les critères de sélection de ces établissements étaient d'ordre technique (potentiel humain suffisant, tonnage bovin minimal) et épidémiologique (représentativité, type et origine de la clientèle...). Trois étapes ont précédé le lancement définitif du réseau d'épidémiosurveillance en abattoir : formation des acteurs, standardisation de la collecte et validation des procédures. Cette surveillance a couvert intégralement les années 1994, 1995 et 1996 et se prolonge actuellement avec le concours de nouveaux abattoirs.

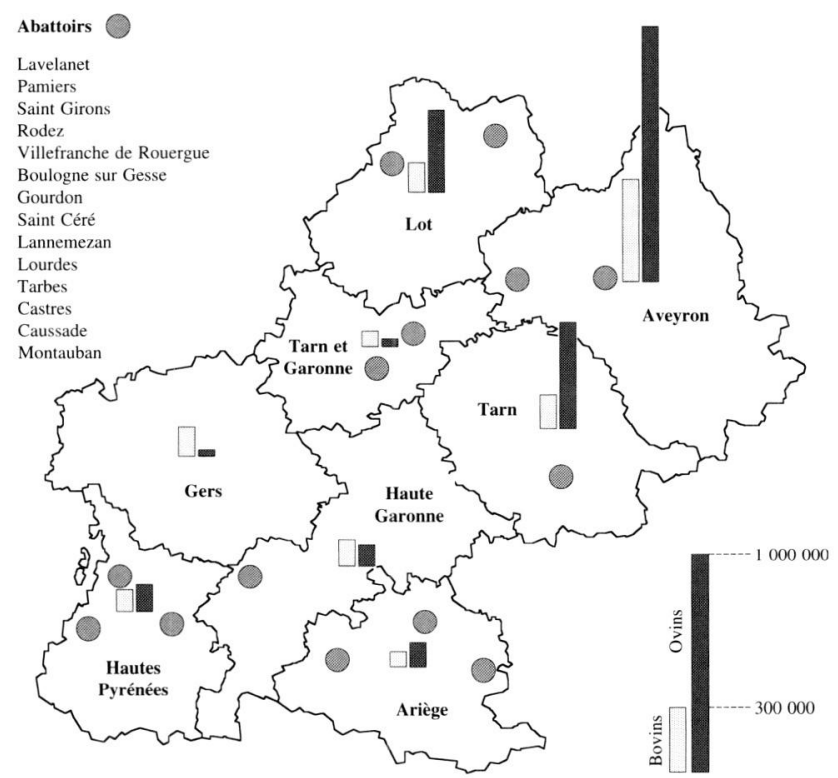

Fig. 1. - Abattoirs du Réseau VEGA et effectifs bovins et ovins départementaux.

\section{CHOIX DES LÉSIONS OBSERVÉES}

La collecte des informations de saisie en abattoir regroupe trois thèmes d'étude: l'hydatidose, la fasciolose et la dicrocœeliose. Le choix de ces affections se fonde sur des critères économiques ou de santé publique et prend en considération trois éléments importants :

- ces pathologies sont crypto-symptomatiques, dans le contexte local,

- les éleveurs ne sont pas informés, par les Services d'Inspection Vétérinaire, des saisies concernant le cinquième quartier (viscères),
- la lutte contre ces parasitoses est très rentable (douves) et/ou confère une excellente garantie contre les risques d'infestation humaine (échinocoque).

\section{BUT ET OBJECTIF}

La démarche d'épidémiosurveillance régionale a pour objectif la diminution des pertes de production et la mâ̂trise des risques sanitaires. A travers la description et l'évaluation de la situation dans le temps et dans l'espace, elle vise à la décroissance du taux de prévalence des parasitoses faisant l'objet de suivis. L'hydatidose bovine s'inscrit dans ce schéma. D'un point de vue économique, elle entraine la saisie d'un certain nombre de viscères (foies, poumons); sous son aspect zoonosique elle est révélatrice de l'existence d'un cycle rural impliquant vraisemblablement d'autres espèces animales, domestiques ou sauvages (ovins, porcins). La démarche d'épidémiosurveillance est donc intéressante. Enfin, l'action du Réseau VEGA permet d'évaluer l'effet de la restitution aux éleveurs d'une information personnalisée dans le cadre de la lutte contre une parasitose grave.

\section{Population CiBle}

La population observée est constituée de bovins adultes provenant des cheptels régionaux. L'effectif total de la région est d'environ 1,3 million de bovins répartis en 31000 étables (source : Chambre régionale d'agriculture Midi-Pyrénées, 1995). L'identification des bovins et la connaissance précise de leur aire d'élevage, grâce aux documents d'accompagnement (cartes vertes annuelles), permettent d'une part d'effectuer un traitement statistique et cartographique des données au plan régional et d'autre part d'informer nominativement chaque éleveur des saisies effectuées sur ses animaux et des risques encourus (Fig. 2). L'absence d'identification généralisée chez les ovins (réforme en cours) et la complexité des circuits commerciaux rendent un tel suivi impossible dans cette espèce.

L'échantillon d'étude est composé de l'ensemble des bovins adultes, issus de la région, passant à l'abattoir.

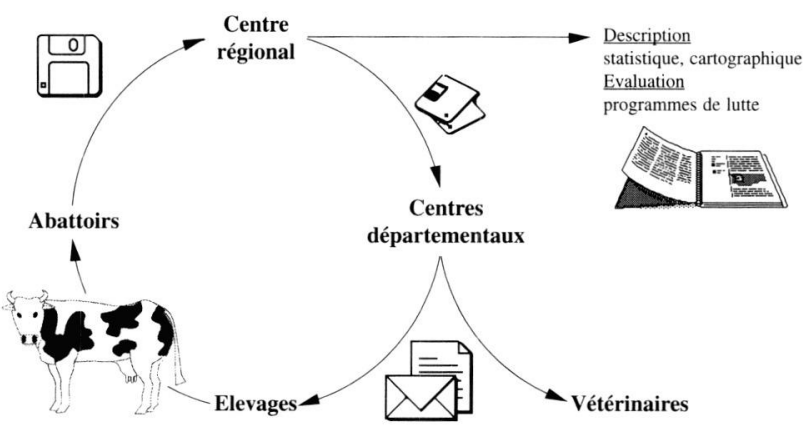

Fig. 2. - Circulation de l'information dans la filière abattoir du Réseau VEGA. 
Ces animaux sont exposés au risque de contamination de par leur âge et la conduite d'élevage, contrairement aux veaux de batteries et aux taurillons d'ateliers d'engraissement. Il s'agit essentiellement de bovins de réforme et de gros bovins de boucherie.

Il n'existe aucun lien entre l'abattoir de destination et l'infestation par des larves de E. granulosus et ce pour deux raisons essentielles : d'une part aucune manifestation clinique n'étant perceptible, le tri a priori des animaux est impossible et d'autre part la saisie n'affectant en rien le prix de la carcasse, aucune concurrence entre abattoirs n'est à craindre. L'échantillon observé dans les abattoirs du Réseau VEGA est donc représentatif des bovins adultes provenant de Midi-Pyrénées.

\section{Traitement statistique}

Une base de données a été développée, en trois modules, permettant la collecte (abattoirs), le traitement (centre régional) et la restitution des informations nominatives aux éleveurs (centres départementaux). L'analyse porte d'une part sur les animaux, de l'autre sur les cheptels. Seuls ont été pris en considération les bovins issus d'étables de la région. Les abattoirs transmettent un fichier d'ensemble comprenant les animaux avec ou sans lésions.

- Taux de prévalence des animaux

Le taux de prévalence des animaux correspond au rapport de bovins porteurs de lésions à l'ensemble des bovins abattus. Les valeurs départementales ainsi obtenues ont un intérêt comparatif du point de vue statistique et géographique. Néanmoins, cette surveillance a une vocation régionale. Les résultats doivent donc refléter une situation globale en s'affranchissant le plus possible des effets "département " et "clientèle d'abattoir". De ce fait, le calcul de la prévalence animale régionale prend en compte la contribution de chaque département au prorata de son effectif de bovins adultes. L'intervalle de confiance est calculé sur la base de la prévalence attendue (études antérieures) et du nombre d'observations effectuées (SVHA, 1981; Chermette, 1983; Maillot, 1989)

- Taux de prévalence des cheptels

L'unité "élevage " conduit au calcul du taux de prévalence des cheptels. Elle offre une vision plus fine de la situation sanitaire, notamment en terme de répartition et d'estimation du nombre de foyers. Elle permet, en outre, de conduire des actions ciblées visant à faire diminuer le risque de contamination.

Si le regroupement des animaux abattus, par cheptel d'origine, ne pose que peu de problèmes, la méconnaissance de la prévalence au sein d'un troupeau impose un choix entre deux attitudes possibles : déterminer $a$ priori le taux d'infestation dans le cheptel ou prendre en considération la distribution de l'âge des animaux en élevage et des animaux abattus. Cette deuxième option parait plus propice au calcul du nombre nécessaire de sujets à observer pour qualifier un troupeau de sain ou d'infesté. Le raisonnement est le suivant : en moyenne, le cheptel bovin régional est constitué de 43 animaux de un à huit ans, soit environ six individus par classe d'âges. Le renouvellement annuel s'effectue en priorité en réformant les animaux les plus âgés et donc potentiellement les plus contaminés. L'examen de six à sept bovins par étable fournit une appréciation correcte de l'infestation du cheptel. Les cas d'échinococcose, dans le contexte local, ne sont effectivement rencontrés que sur des animaux âgés. Le calcul de l'indicateur fait donc intervenir le nombre d'animaux abattus dans l'année. Un cheptel est déclaré positif si un animal au moins est porteur de lésions. Le taux de prévalence annuelle des cheptels est le rapport du nombre d'élevages positifs ayant abattu $n$ bovins dans l'année, à l'ensemble des élevages ayant abattus $n$ bovins dans l'année, $n$ variant de un à huit. En pondérant les résultats départementaux par le nombre d'élevages de chaque département, il est possible d'estimer le taux de prévalence de l'hydatidose des cheptels en Midi-Pyrénées.

\section{RÉSULTATS}

\section{TAUX DE PRÉVALENCE DES ANIMAUX}

es trois années de collecte ont permis de répertorier 134 cas d'échinococcose bovine répartis en 131 cheptels (Fig. 3). Les observations ont porté sur 43148 bovins, soit un taux de prévalence appa-

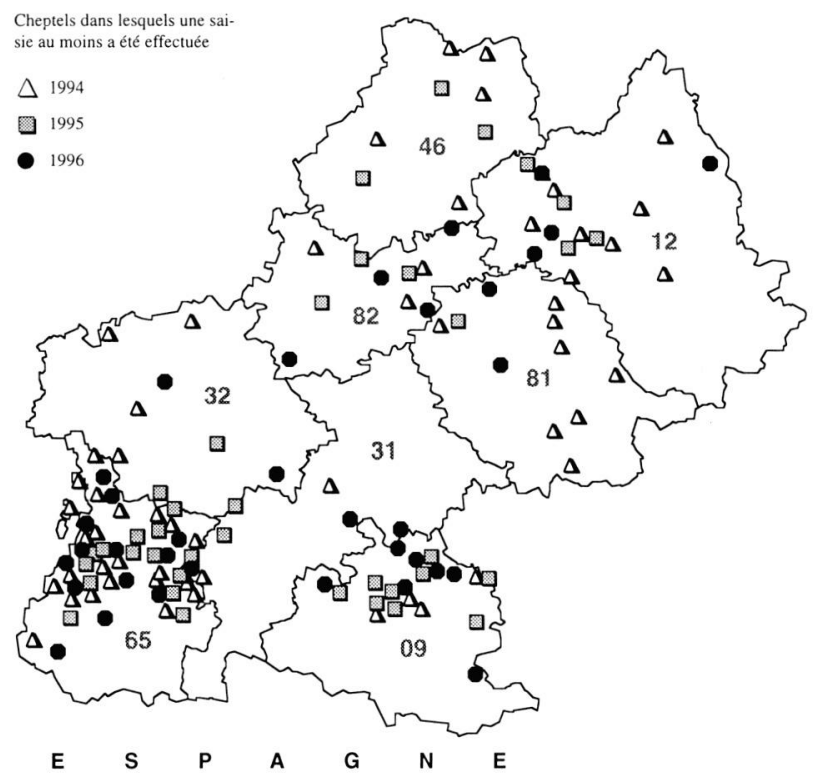

Fig. 3. - Répartition des saisies pour hydatidose bovine en MidiPyrénées, durant la période 1994-1996, répertoriées dans les abattoirs du Réseau d'épidémiosurveillance VEGA. 
rente de 0,31\%, durant la période 1994-1996. L'évolution de ce taux brut est sensible au cours des trois années, 0,55\% en 1994, 0,32\% en 1995 et 0,17\% en 1996. En pondérant ces résultats par l'effectif de chaque département, on obtient une meilleure estimation du taux de prévalence régionale annuelle : 0,69\% en $1994,0,26 \%$ en 1995 et $0,16 \%$ en 1996 et un taux de prévalence de $0,28 \% \pm 0,05$, sur la période totale (Fig. 4, 5 et 6).

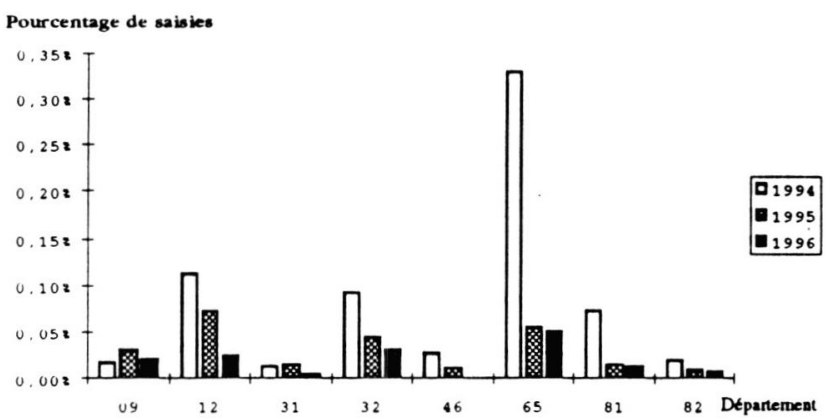

Fig. 4. - Évolution des saisies pour échinococcose, par département et par année.

\section{TAUX DE PRÉVALENCE DES CHEPTELS}

Le regroupement des animaux par cheptel d'origine offre une base d'étude de 19108 élevages, soit plus de $60 \%$ des troupeaux de la région. La mise en œuvre de la méthode de calcul précédemment décrite conduit à établir, pour chaque année, une courbe de prévalence apparente en fonction du nombre d'animaux abattus par cheptel (Fig. 7). Eu égard aux caractéristiques de l'échantillon observé, la meilleure estimation de l'indicateur se situe, comme cela était attendu, à six/sept bovins abattus. Les trois courbes passent effectivement par un maximum pour ces valeurs. La moyenne des données annuelles, pour chaque classe d'abattage, conduit à l'établissement d'une courbe sur la période triennale. L'estimation, pour les cheptels, du taux de prévalence est de 2,5\% $\pm 1,0 \%$.

\section{DISCUSSION}

\section{PertinenCe DeS RÉSUltats}

T es données régionales obtenues recoupent largement les informations recueillies en 1989 lors de l'enquête nationale effectuée à la demande du ministère de l'Agriculture (Fig. 8, Tableau I). En matière d'hydatidose bovine, le département des Hautes-Pyrénées est manifestement le plus atteint de la région. Les valeurs obtenues restent néanmoins bien en deçà du niveau d'infestation du Sud-Est de la France.

Dans de nombreux départements, une certaine dissociation entre les taux d'infestation bovine, ovine et por-

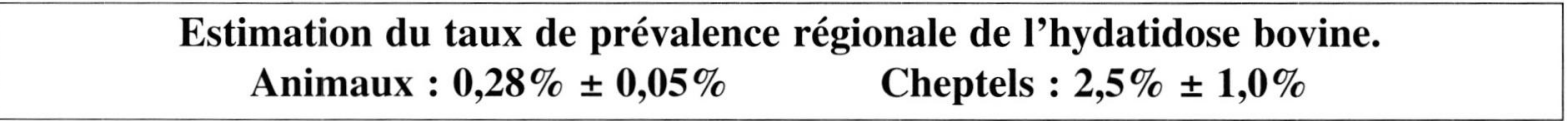

Département

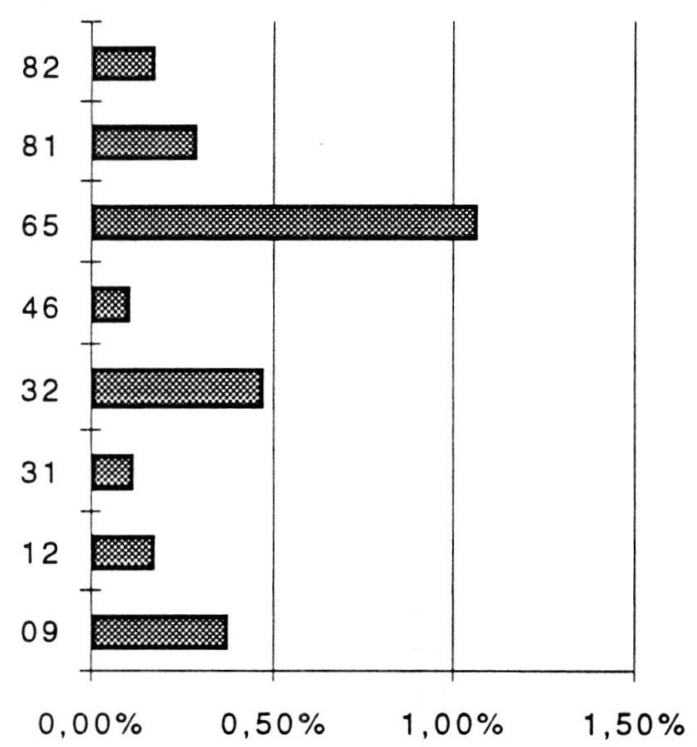

Fig. 5. - Estimation des taux de prévalence départementaux de l'hydatidose bovine en Midi-Pyrénées.

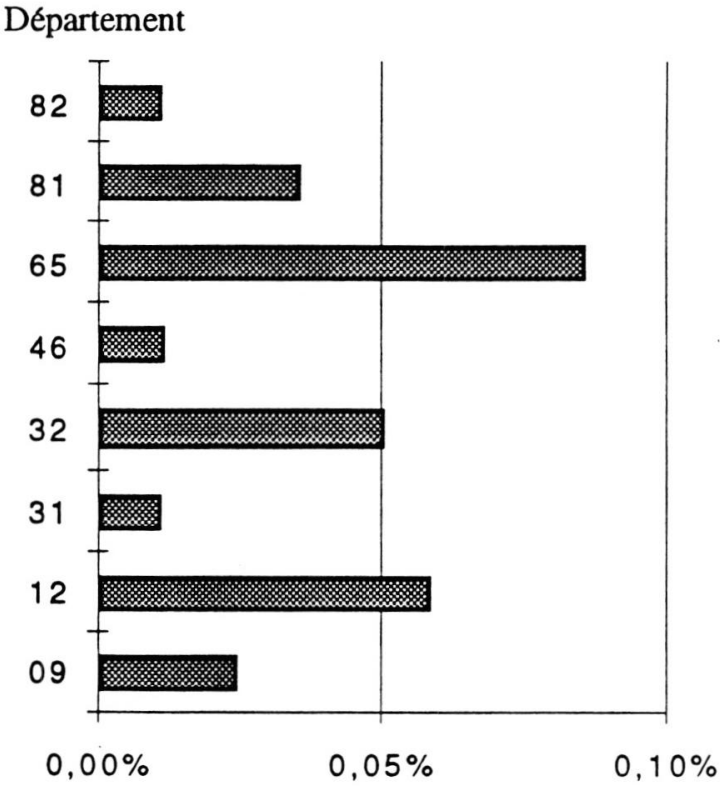

Fig. 6. - Contributions départementales au calcul du taux de prévalence régionale de l'hydatidose bovine en Midi-Pyrénées. 


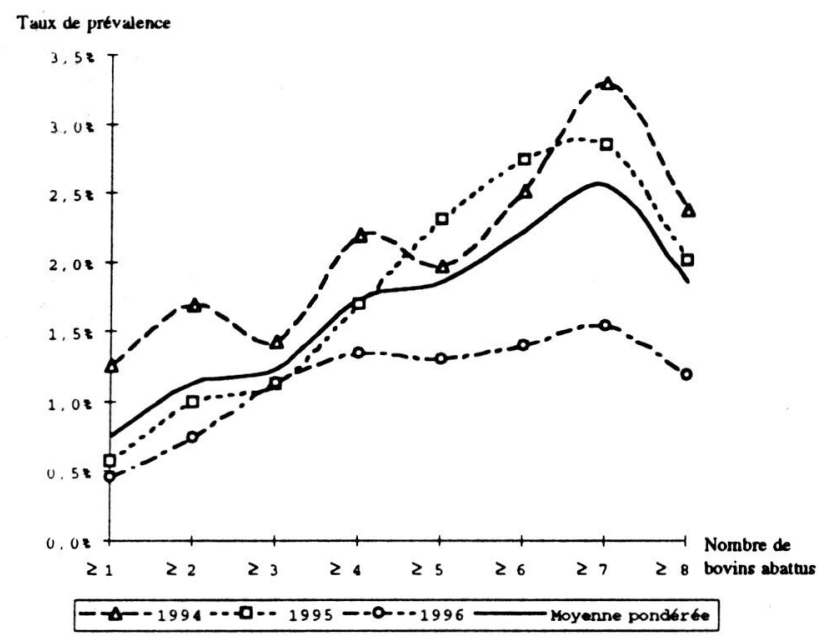

Fig. 7. - Estimation du taux de prévalence de l'hydatidose des cheptels en fonction du nombre de bovins observés.

\begin{tabular}{lcc}
\hline & \multicolumn{2}{c}{ Taux de prévalence estimé } \\
\cline { 2 - 3 } & $\begin{array}{c}\text { Enquête 1989 } \\
\text { nationale }\end{array}$ & $\begin{array}{c}\text { Enquête 94/96 } \\
\text { régionale }\end{array}$ \\
\hline Ariège (09) & $0,12 \%$ & $0,37 \%$ \\
Aude (11) & $0,06 \%$ & $0,17 \%$ \\
Aveyron (12) & $0,12 \%$ & $0,11 \%$ \\
Haute-garonne (31) & $0,04 \%$ & $0,46 \%$ \\
Gers (32) & $0,34 \%$ & $0,10 \%$ \\
Lot (46) & $1,09 \%$ & $1,06 \%$ \\
Pyrénées-Atlantiques (64) & $1,03 \%$ & $0,28 \%$ \\
Hautes-Pyrénées (65) & $0,13 \%$ & $0,16 \%$ \\
Tarnén (81) & $0,46 \%$ & \\
Tarn-et-Garonne (82) & &
\end{tabular}

Tableau I. - Estimation des taux de prévalence de l'hydatidose bovine.

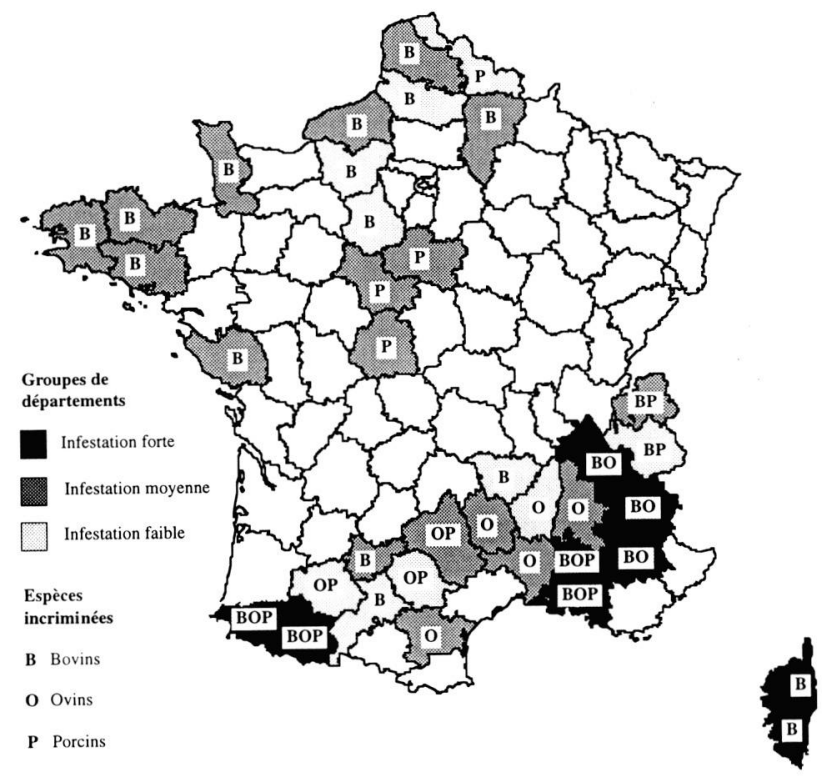

Fig. 8. - Prévalence de l'hydatidose sur les animaux d'abattoir et espèces principalement concernées, en 1989.

cine a été révélée par l'enquête nationale. Ceci s'explique vraisemblablement par la spécialisation sectorielle des productions, l'absence de corrélation entre les conduites d'élevages dans ces différentes espèces et la circulation de différentes souches d'échinocoques. En effet, la forte densité ovine ne permet pas d'expliquer le taux d'hydatidose bovine (l'Aveyron est le premier département ovin de France). Par contre, le lien avec la transhumance, ovine en particulier, semble plus étroit (Tableau II). Le coefficient de corrélation entre ce taux de prévalence et le nombre de cheptels ovins transhumants est de 0,87 . Il n'est que de 0,52 si l'on considère le nombre de cheptels bovins transhumants.
Taux de prévalence hydatidose bovine
Nombre de cheptels ovins transhumant

Chambre d'Agriculture
Nombre de cheptels bovins transhumant

Source

Réseau VEGA

Chambre d'Agriculture

\section{Ariège (09)}

Aude (11)

Aveyron (12)

Haute-garonne (31)

Gers (32)

Lot (46)

Pyrénées-Atlantiques (64)

Hautes-Pyrénées (65)

Pyrénées-Orientales (66)

Tarn (81)

Tarn-et-Garonne (82)

$0,37 \%$
$0,17 \%$
$0,11 \%$
$0,46 \%$
$0,10 \%$
$1,06 \%$
$0,28 \%$
$0,16 \%$

Corrélation : Taux de prévalence-transhumance ovine $=0,87 . \mathrm{R}^{2}=0,75$.

Corrélation : Taux de prévalence-transhumance bovine $=0,52 \cdot \mathrm{R}^{2}=0,28$.

Tableau II. - Taux de prévalence de l'hydatidose bovine et transhumance dans le sud-ouest de la France. 
Les facteurs favorisant la circulation parasitaire sont essentiellement la consommation de cadavres par les chiens de berger et le mélange de nombreux troupeaux (Euzeby, 1971). Ce dernier élément pourrait expliquer partiellement les différences de taux de prévalence entre les deux massifs. La transhumance pyrénéenne est presque exclusivement intradépartementale (550000 ovins) contrairement au flux migratoire alpin (640 000 ovins) caractérisé par un intense brassage d'animaux d'origines départementales et régionales variées. D'autres éléments sont à prendre en compte pour expliquer les différences départementales en MidiPyrénées, comme la présence d'une faune sauvage sensible et le passage d'animaux espagnols sur les estives, l'échinococcose-hydatidose étant enzootique en Espagne (Perez, 1991).

\section{ÉVOLUTION DU TAUX DE PRÉVALENCE}

En reprenant la même méthode de calcul et en l'appliquant aux données obtenues en 1989, le taux de prévalence régionale de l'échinococcose bovine aurait été de 0,23\%, comparable à celui de 1995. Durant la période 1994-1996 trois phénomènes importants peuvent être notés :

- les taux de prévalence des animaux et des cheptels diminuent fortement,

- la décroissance n'est pas constante au fil des années,

- la chute de la prévalence des animaux précède d'un an celle des cheptels (Fig. 9).

Ces constatations s'expliquent par l'action combinée de la sensibilisation des éleveurs par le retour d'information personnalisé et par une élimination légèrement accrue de bovins âgés, en 1994 et 1995, conséquemment à la crise de l'encéphalopathie spongiforme bovine.

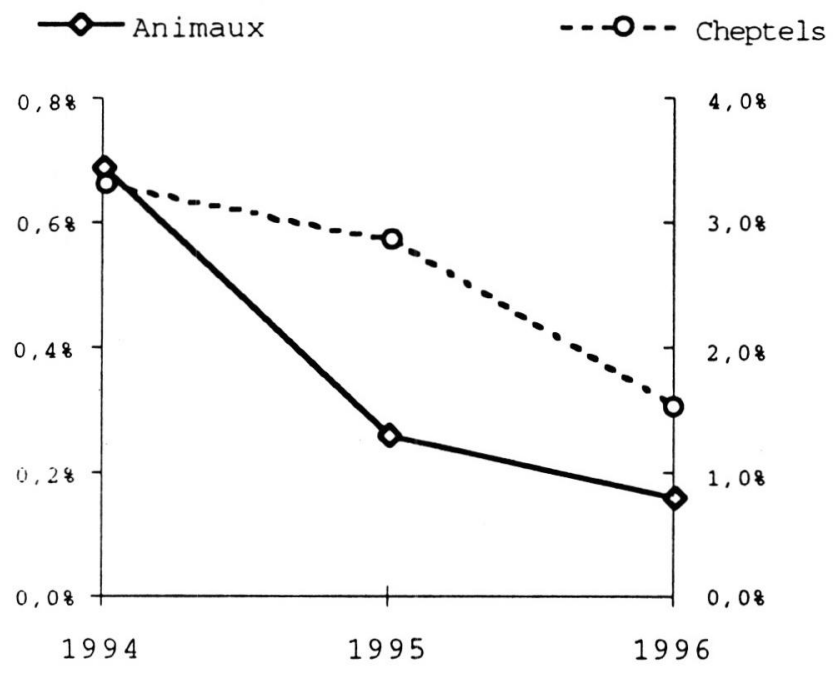

Fig. 9. - Évolution des taux de prévalence de l'hydatidose en MidiPyrénées.

\section{BILAN DES ENQUÊTES LOCALES}

Les données publiées sont en nombre assez restreint et portent davantage sur les ovins que sur les bovins. La mise en œuvre d'enquêtes est étroitement liée à la sensibilisation des acteurs sanitaires et se retrouve, tout naturellement dans le sud-est de la France du fait de l'importance du nombre de cas d'hydatidose humaine, liés à certaines pratiques culturelles ou religieuses comme l'Aïd el kebir (Dumon, 1984; Charnine, 1986).

\section{- Sud-Est}

La région Provence-Alpes-Côte d'Azur a fait l'objet de diverses études, essentiellement dans l'espèce ovine. Certaines enquêtes ont été effectuées en abattoirs, à la demande de l'OMS, par les Directions des Services Vétérinaires (1980/1981) ou à l'initiative de Dumon (Dumon, 1981). D'autres, non plus lésionnelles mais sérologiques, ont été menées par le Laboratoire de Parasitologie de la Faculté de Médecine de Marseille et la Direction des Services Vétérinaires des Bouches-du-Rhône (Dumon, 1984). Nozais a étudié la situation de l'hydatidose ovine sur le pourtour méditerranéen, durant plusieurs années jusqu'en 1989 (Nozais, 1989). Selon les auteurs et les départements d'origine des moutons, le taux de prévalence varie de 1 à $81 \%$, maximum atteint en Corse du Sud (Dumon, 1984; Blisson, 1987; Lauzier, 1987).

Par ailleurs, une enquête menée en avril 1985 sur une population de chiens de berger dans les Bouches-duRhône a révélé que $30 \%$ des chiens évacuaient au moins un exemplaire de E. granulosus après une vermifugation au bromhydrate d'arécoline (Casset, 1985). Enfin, seul un bilan comparatif de l'atteinte parasitaire des différentes espèces animales abattues à l'abattoir de Gap en 1981 nous fournit une référence bovine (SVHA, 1981). Cependant, il ne s'agit non plus d'une estimation départementale mais de l'image d'une clientèle d'abattoir (Tableau III).

- Sud-Ouest

Seul le département des Pyrénées-Atlantiques a effectué dans le passé (1980-1981) une étude de prévalence de l'hydatidose ovine, révélant une infestation de 15 à 20\% sur les animaux abattus (SVHA, 1981). Le statut parasitaire de la région Midi-Pyrénées n'était connu qu'à

\begin{tabular}{lccc}
\hline Espèce animale & $\begin{array}{c}\text { Animaux } \\
\text { abattus }\end{array}$ & $\begin{array}{c}\text { Nombre de cas } \\
\text { d'hydatidose }\end{array}$ & Pourcentage \\
\hline Ovins & 41598 & 233 & 0,56 \\
Bovins & 2118 & 24 & 1,13 \\
Porcins & 14493 & 96 & 0,66 \\
Équins & 165 & 3 & 1,82 \\
\hline
\end{tabular}

Tableau III. - Prévalence des différentes espèces à l'abattoir de Gap, 1981. 
travers l'enquête nationale. De nombreuses similitudes existaient entre cette région et Rhône-Alpes, en termes de taux d'infestation et d'espèces animales incriminées. Ces deux régions, de huit départements chacune, se situent au second rang derrière Provence-Alpes-Côte d'Azur (Maillot, 1989). La surveillance épidémiologique du Réseau VEGA permet de confirmer en grande partie l'étude de 1989 et introduit la notion de foyer. Cet indicateur est nettement moins sensible au biais de recrutement et rend possible une lutte raisonnée.

\section{CONNAISSANCES ACQUISES}

Il est intéressant de constater que dans le contexte régional, la pression parasitaire est trop faible pour permettre le développement de foyers fortement contaminés. Les 134 saisies sont dispersées en 131 cheptels. La contagiosité au sein des troupeaux semble faible en Midi-Pyrénées. Cet élément est à rapprocher des bonnes pratiques d'élevage locales :

- limitation de la consommation d'abats crus par les chiens de fermes,

- contrôle du brassage des espèces animales, en particulier sur les estives,

- maintien des chiens hors des troupeaux.

L'infestation bovine doit être considérée comme un révélateur de l'existence d'un cycle rural affectant principalement les ovins. En effet, la contamination des chiens par des viscères de bovins est, dans le contexte régional, pratiquement impossible.

Le typage des larves d'échinocoques permettrait de vérifier à quelle souche appartiennent les larves saisies à l'abattoir sur les bovins. La parenté génétique des souches ovines, bovines et humaines ayant été, par ailleurs, rapportée par de nombreux auteurs (SilesLucas, 1996).

Du point de vue de l'inspection vétérinaire en abattoir, il semblerait qu'en Midi-Pyrénées les lésions pulmonaires soient nettement plus fréquentes que les lésions hépatiques chez les bovins et les ovins, contrairement à ce qui est classiquement décrit. Ce constat n'est pas sans rappeler la localisation préférentielle des larves hydatiques chez l'enfant, par opposition à l'adulte (Akcali, 1991; Bourée, 1991; Sagne, 1992; Utrilla, 1991 ; Xu, 1991).

\section{PRÉVALENCE DE L'HYDATIDOSE HUMAINE}

Au plan de la santé publique, le nombre de cas autochtones, pour l'essentiel d'origine ovine, serait d'environ 300 pour la France métropolitaine Corse comprise, soit 0,5 cas pour 100000 habitants (Sagne, 1992). En fait, ces valeurs sont entachées d'une large incertitude puisqu'aucune enquête nationale n'a été menée (l'hydatidose n'étant pas une maladie à déclaration obligatoire) et que la durée de la phase asymptomatique empêche de déterminer avec précision la date de l'infestation. Ces estimations s'appuient sur des données ponc- tuelles, notamment celles collectées par H. Dumon dans les hôpitaux de la région marseillaise et de la Corse, de 1966 à 1970 puis de 1971 à 1978. Elle a permis de révéler l'existence d'un foyer corse, dix cas pour 100000 habitants et d'un foyer provençal, 4,5 cas pour 100000 habitants (Dumon, 1981; Dumon, 1984). Ou encore, en région parisienne à l'hôpital SaintAntoine, six patients sur 17 opérés en 1980 n'avaient jamais quitté l'Ile-de-France (Dry, 1980).

Des bilans plus larges effectués en 1985 et 1987, dans 41 et 33 laboratoires de parasitologie des CHU/CHR ont permis de répertorier respectivement 685 et 458 cas d'échinococcose, dont 19 puis neuf en Midi-Pyrénées (source: Bulletin Épidémiologique Hebdomadaire $n^{\circ} 35$, 1990).

En 1995, environ 30 cas " autochtones " ont été rapportés par les dix principaux centres hospitaliers de la région. Rapportés à la population régionale, environ 2430000 personnes, le taux d'infestation humaine serait de 1 à 1,5 cas pour 100000 habitants. Une certaine cohérence apparaît dans ce résultat qui est supérieur à la moyenne nationale mais inférieur aux valeurs des zones d'endémie. La corrélation attendue entre le taux d'infestation humaine et l'atteinte du cheptel local semble se vérifier. Néanmoins, le caractère autochtone est souvent difficile à vérifier puisque la contamination chez l'enfant n'est généralement détectée qu'à l'âge adulte. Une certaine réserve doit donc entourer ces résultats.

\section{POURSUITE DE LA DÉMARCHE}

L'information des éleveurs reste, dans le contexte pyrénéen, la méthode la plus sûre pour rompre le cycle parasitaire. Néanmoins, les mesures de prophylaxie sanitaire et médicale ne sont que partiellement appliquées. Un traitement obligatoire et systématique des chiens accompagnant les animaux en estive, à la montée et à la descente, devrait être envisagé. Seule une action conjointe alliant circulation des données, sensibilisation de tous les partenaires de l'élevage (vétérinaires, techniciens...) mais également responsabilisation des producteurs, pourra entrainer une diminution franche et durable du risque parasitaire. Une telle démarche, s'étendant aux écoles, a largement été couronnée de succès en Nouvelle-Zélande (Schwabe, 1969). Par ailleurs, l'extension de la surveillance aux moutons est envisageable dans un avenir proche, eu égard à l'adoption d'une nouvelle réglementation en matière d'identification ovine.

\section{CONCLUSION}

es structures d'abattage et d'équarrissage, en France, font de l'hydatidose bovine l'un des révélateurs du cycle parasitaire rural. Son lien étroit avec la transhumance semble se manifester et fait des contreforts pyrénéens le secteur le plus touché de 
Midi-Pyrénées. Néanmoins, l'infestation est ubiquitaire et concernerait environ $0,3 \%$ des bovins et $2,5 \%$ des cheptels. Ces estimations placent cette région au deuxième rang national derrière Provence-Alpes-Côte d'Azur.

Un programme de lutte efficace se doit d'intégrer la vermifugation systématique des chiens à la descente d'estive au même titre que les chiens présents dans les foyers. Bien que manquant de recul, une évolution favorable et progressive a été enregistrée de 1994 à 1996. Le retour d'information aux éleveurs semble contribuer à diminuer le risque sanitaire.

Le nombre de cas humains autochtones est faible mais non nul. Il est révélateur, comme partout ailleurs, de comportements permissifs voire irresponsables, fondés sur la méconnaissance du cycle parasitaire, et dans tous les cas du non-respect des règles élémentaires d'hygiène. L'existence de l'Association Internationale d'Hydatidologie, le nombre de publications consacrées à ce sujet mais également les directives de l'OMS doivent nous rappeler que le maintien d'un bon statut sanitaire est fragile, particulièrement en période de restriction économique.

\section{RÉFÉRENCES}

Plusieurs références sont issues de

Archivos de la hydatidosis vol. XXX. XV Extraordinary Congress for the Celebration of the 50 years of Association International Hydatidosis. Rome, November 4-8, 1991. F. de Rosa Ed. JL., CIC. Rome, Milan, Paris, New York.

Akcali Y., Kahraman C., Tasdemir K., Dural K., Köseahmetoglu M., Ceberrut K. \& Oguzkaya F. Surgical treatment of pulmonary hydatid disease. A 12 year experience with 197 cases. Archivos de la hydatidosis, 1991, XXX, 691-695.

Blisson G. \& CASSET I. Développement d'un foyer d'hydatidose dans le sud-est de la France. Dépistage du ténia chez le chien. Revue de Médecine Vétérinaire, 1987, 138, 27-31.

Bourée P. \& HeIderijk P. Les échinococcoses. Revue Française des Laboratoires, 1991, 228, 19-22.

Casset I. L'hydatidose dans les Bouches-du-Rhône, enquête sur l'infestation des chiens de berger. Thèse Doct. Méd. Vét., Toulouse, 1985, 53 pp.

Charnine F. Le kyste hydatique du foie : A propos de 127 cas opérés au CHU de Monastir (1979-1984). Thèse Doct. Méd., Toulouse, 1986.

Chermette R. Importance et situation actuelle des échinococcoses larvaires en France ( $1^{\text {ère }}$ partie) : L'hydatidose, conséquences pratiques en médecine vétérinaire. Le Point Vétérinaire, 1983, 14, 15-24.

Cruz PaIz J. Hidatidosis hepatica, nuestra experiencia. Archivos de la hydatidosis, 1991, XXX, 1083-1091.

Dry, Leynadier, Luce, Abrego, Huguier \& Huget. Diagnostic du kyste hydatique par le test de dégranulation. Société Médicale des Hôpitaux de Paris. Compte rendu des sociétés savantes, 1980, 30, 31, 32.

Dumon H., Raoult D., Ramahandridona N. \& Quilici M.
L'hydatidose en Provence et en Corse. Enquête épidémiologique. Bulletin de la Société de Pathologie Exotique, 1981, 74, 96-100.

Dumon H., Toga I., Gambarelli F., Faugere B., Franck J., Dunan S., Mary Ch., Quilici M. \& Bailenger J. Épidémiologie de l'échinococcose hydatique en France. Réflexion à propos de l'endémie dans les foyers de Corse, de Provence et du sud-ouest. Bulletin de la Société Française de Parasitologie, 1984, 2, 69-73.

EuzeBy J. Les échinococcoses animales et leurs relations avec les échinococcoses de l'homme. Vigot Frères Éditeurs, Paris, 1971.

EuzeBy J. La spécificité parasitaire: incidences sur l'étiologie et sur l'épidémiologie des parasitoses humaines d'origine zoonosique. Éd. Fondation Marcel Mérieux, Lyon, 1997.

Gusbi A.M., Awan M.A.Q. \& BeEsley W.N. Echinococcosis in Libya. IV. Prevalence of hydatidosis (Echinococcus granulosus) in goats, cattle and camels. Annals of Tropic Medecine and Parasitology, 1990, 84, 477-482.

LAUzier P. Échinococcose à Echinococcus granulosus: Rappels épidémiologiques, enquête dans un foyer des HautesAlpes. Thèse Doct. Méd.Vét., Lyon, 1987, 131 p

Maillot E., Soulé C. \& Fabien J.F. Enquête échinococcosehydatidose dans les abattoirs, Direction Générale de l'Alimentation, Paris, 1989.

Mate T. Programa de control de hidatidosis-indadores (España). Archivos de la hydatidosis, 1991, XXX, 85-98.

Navarrete I., Serrano F., Perez E., Brena M., Morales I. \& GIL F. Study of prevalence of canine echinococcosis in Extremadura: Possible influence over ovine production. Archivos de la hydatidosis, 1991, XXX, 1253-1260.

Nozais J.P. L'hydatidose dans le bassin méditéranéen. Historique, répartition actuelle. Médecine et Maladies Infectieuses, 1989, 19, 439-443.

Perez Gallardo M. \& Quintela Bereciartua E. La hidatidosis en España. I Congreso International de las Associaciones Sud-Occidental-Europeas de Parasitologia. Parasitologia Ed S. Mas-Coma et coll., 1991, 333-334.

SAGNE M.C. Hydatidose de l'enfant et de l'adulte, mise à jour bibliographique, évaluation d'un kit ELISA. Thèse Doct. Méd., Toulouse, 1992, 127 p.

SCHWABE C.W. Veterinary medecine and human health. 3rdedition. Williams and Wilkins, Baltimore, London, 1984.

Siles-Lucas M., Benito M.C. \& Cuesta-Bandera C. Echinococcus granulosus: genomic and isoenzymatic study of Spanish strains isolated from different intermediate hosts. Veterinary parasitology, 1996, 63, 273-282.

SVHA. Enquête épidémiologique du Service vétérinaire d'hygiène Alimentaire, Direction de la Qualité, Ministère de l'Agriculture, Paris, 1981.

Utrilla J.G., Eyre F.P., Muguerza R., Alami H. \& Bueno J. Hydatidosis en la infancia. Archivos de la hydatidosis, 1991, XXX, 721-730.

$\mathrm{XU}_{\mathrm{U}}$ M.Q. Improvement of surgical treatment of hydatid disease of the liver (analysis of 1319 operations). Archivos de la hydatidosis, 1991, XXX, 623-632.

Reçu le 21 juin 1997 Accepté le 4 novembre 1997 